\title{
Erratum to: Multi-camera systems use for dental arch shape measurement
}

\author{
Aleš Procházka ${ }^{1}$ - Magdaléna Kašparová ${ }^{3}$. \\ Mohammadreza Yadollahi $^{1}$. Oldřich Vyšata ${ }^{2}$. \\ Lucie Grajciarová ${ }^{1}$
}

Published online: 16 December 2016

(C) Springer-Verlag Berlin Heidelberg 2016

\section{Erratum to: Vis Comput (2015) 31:1501-1509 DOI 10.1007/s00371-014-1029-z}

In reference 34 the family name of the third author was misspelled by error. The correct wording of reference 34 is given below.

34. Popielski, P., Wrobel, Z., Koprowski, R.: Object Detail Correspondence Problem in Stereovision. In: Pietka, E., Kawa, J., Wieclawek, W. (eds.) Information Technologies in Biomedicine. Springer Int. (2014)

The online version of the original article can be found under doi:10.1007/s00371-014-1029-z.

\footnotetext{
Aleš Procházka

A.Prochazka@ieee.org

Magdaléna Kašparová

Magdalena.Kasparova@fnmotol.cz

Mohammadreza Yadollahi

Yadollao@vscht.cz

Oldřich Vyšata

Vysatao@gmail.com

Lucie Grajciarová

Lucie.Grajciarova@vscht.cz

1 Department of Computing and Control Engineering, Institute of Chemical Technology, Technická 5, 16628 Prague 6, Czech Republic

2 Department of Neurology, Faculty of Medicine in Hradec Králové, Charles University, Sokolská Street 581, 50005 Hradec Kralove, Czech Republic

3 Department of Stomatology, 2nd Medical Faculty, Charles University, V Úvalu 84, 15006 Prague 5, Czech Republic
} 